\title{
Error Minimization in Localization of Wireless Sensor Networks using Ant Colony Optimization
}

\author{
S. Sivakumar, $\mathrm{PhD}$ \\ Assistant Professor (SG) \\ Department of Information Technology \\ PSG College of Technology \\ Coimbatore, India
}

\author{
R. Venkatesan, PhD \\ Professor and Head Department of Computer \\ Science \& Engineering \\ PSG College of Technology \\ Coimbatore, India
}

\begin{abstract}
Localization is considered to be one of the most significant research issues in a Wireless Sensor Networks (WSN). The major objective of localization is in estimating the physical co-ordinates of a group of sensor nodes distributed along its sensing field. The location information plays a prominent role for coverage and deployment of sensor nodes, routing and target tracking applications as well. The objective of this work is to determine the location of the sensor nodes with minimal error and high precision. Initially, the location estimation for the sensor nodes is carried out by using Mobile Anchor Positioning with Mobile Anchor \& Neighbor (MAP-M\&N), a range-free localization method. As the anchors move through the network, they broadcast their location as beacon packets. The sensor nodes use the location information of beacon packets obtained from mobile anchors and also the location packets from neighboring nodes to calculate their location. In order to get better location accuracy, Ant Colony Optimization with Mobile Anchor Positioning (ACO-MAP) is proposed for localization in WSN. The goal of this work is to compare the performance of ACO-MAP and MAP-M\&N approaches by taking into consideration Root Mean Square Error (RMSE) as the performance metric. Simulation results reveal the fact that, Ant Colony Optimization with Mobile Anchor Positioning (ACO-MAP) is efficient to minimize the localization error when compared to MAP-M\&N algorithm.
\end{abstract}

\section{General Terms}

Localization in Wireless Sensor Networks.

\section{Keywords}

Localization, Mobile Anchor, Wireless Sensor Network, Ant Colony Optimization, Root Mean Square Error;

\section{INTRODUCTION}

A Wireless Sensor Network (WSN) is a class of ad hoc network that consists of independent sensors with low cost, low energy sensing devices, which are connected by wireless communication links. Sensor nodes are tiny in size and possess limited resources namely processing, storage, sensing and communication [1]. In general these sensors are deployed in large numbers over the region of interest for object monitoring and target tracking applications. The densely deployed sensors are expected to know their spatial coordinates for effective functioning of WSNs. Location awareness plays an important role in high-level WSN applications like locating an enemy tank in a battlefield and locating a survivor during a natural calamity and in certain low-level network applications like geographic direction finding and data centric storage.

Localization is a fundamental problem which can be defined as the process of finding the position of the sensor nodes or determination of spatial co-ordinate of the sensor nodes.
Localization is particularly important [2] when there is an ambiguity on the exact location of fixed or mobile devices. Localization means the method of making every sensor node in the sensor network to be aware of its geographic position [3]. The usual solution is to equip each sensor with a GPS receiver that can provide the sensor with its exact location. As WSNs normally consist of a huge number of sensors, the use of GPS will not be a cost-effective solution and also makes the sensor node bulkier [4]. GPS has inadequate functionality as it works only in open fields and cannot function in underwater or indoor environments. Therefore, WSNs are required of some alternative means of localization.

Currently the existing non-GPS based sensor localization algorithms [5] are classified as range-based or range-free. Range-based localization schemes rely on the use of absolute point-to-point distance or angle estimate between the nodes to determine the position of unknown sensor nodes using some location-aware nodes. Location-aware nodes are also called as anchors. Typical range-based localization techniques used are Received Signal Strength Indicator (RSSI) [6], Time Difference of Arrival (TDoA) [7], Time of Arrival (ToA) [8], and Angle of Arrival (AoA) [9]. Depending on the signal feature used, the position estimation is found using geometrical approaches such as Triangulation, Trilateration or Multilateration. Range-based methods give fine-grained accuracy but the hardware used for such methods are expensive. In range-based mechanisms, the nodes obtain pair wise distances or angles [10] with the aid of extra hardware providing high localization accuracy. Due to cost, the use of range-based methods will not be preferred.

Range-free or proximity based localization schemes rely on the topological information, e.g., hop count and the connectivity information, rather than range information. Range-free localization schemes may or may not be used with anchors or beacons. A range-free localization scheme does not involve in the use of complex hardware and are cheaper when compared to range-based schemes. Range-free methods use the content of messages from anchor nodes and other nodes to estimate the location of non-anchor sensor nodes. Centroid Algorithm [11] and Distance Vector Hop (DV-Hop) method [12] are certain range-free algorithms. Range-free algorithms sometimes use mobile anchors [13] for localization. Rangefree algorithms are not costly but they provide coarse-grained accuracy. Range-free schemes provide lower localization accuracy at lower cost.

Localization in Wireless Sensor Networks is intrinsically an unconstrained optimization problem [14]. Evolutionary algorithms are local search methods, capable of efficiently solving complex constrained or unconstrained optimization problems. 
The rest of the paper illustrates the related research work in this area, elaborates the Mobile Anchor Positioning method and the proposed approach namely, Ant Colony Optimization with Mobile Anchor Positioning (ACO-MAP) and compares the performance of this proposed approach with an existing algorithm namely MAP-M\&N.

\section{RELATED WORK}

W-H Liao et al. [15] proposed an algorithm (Mobile Anchor Positioning) in which each sensor node receives beacons (messages containing location information) in its receiving range from the moving anchor as the anchor moves around the sensing field. Among the received beacons, the sensor node selects the farthest two beacons. The node constructs two circles with each chosen beacon as center. The radius of the circle is the communication range of the sensor node. It determines the intersection points of the two circles. Out of the two points, one is chosen to be the location of the sensor node based on a decision strategy.

Kuo-FengSsu et al. [16] presented a range-free algorithm, which uses the following conjecture. A perpendicular bisector of a chord passes through the centre of the circle. When there are two chords of the same circle, their perpendicular bisectors will intersect at the centre of the circle. A mobile anchor moves around the sensing field broadcasting beacons. Each sensor node chooses two pairs of beacons and constructs two chords. The sensor node assumes itself as the centre of a circle and determines its location by finding the intersection point of the perpendicular bisectors of the constructed chords.

Baoli Zhang et al. [17] proposed a range-free algorithm, which works as follows. The trajectories of the mobile anchor are in such a way that it moves in a straight line. As it moves, it periodically broadcasts its location to the sensor nodes. A sensor node selects four beacons among all collected beacons. The first group (two beacons) is the location of the mobile anchor node when it first enters the communication range of the sensor node. The second group is the location of the mobile anchor node when it second enters the communication range of the sensor node. After these positions and the communication range are obtained, four circles are constructed with the chosen four points as centers. Four intersection points $s_{1}, s_{2}, s_{3}, s_{4}$ of the circles are calculated. Then using the centroid formula on the four intersection points, the position of the sensor node is calculated.

Wenwen $\mathrm{Li}$ et al. [18] proposed the Genetic algorithm for localization of the sensor nodes and constructed the solution space, coded the solutions, formulated the fitness function and appropriate selection mechanism to choose the parents for the next generation. The reproduction operation on the individuals is further performed and the solution is obtained with high accuracy. The above genetic algorithm approach gives good localization accuracy but the solution space is very huge. The algorithm has to search a large number of solutions in each of the iterations or the number of iterations will be large. When the area of the sensing field increases, the computation involved also increases.

The first three approaches have advantages - Like, they do not require additional hardware and depend only on messages passed but they are coarse-grained i.e. their accuracy will not be very high.

Gopakumar et al. [19] proposed the swarm intelligence based approach for localization of the sensor nodes for this nonlinear optimization problem. The objective function chosen is the mean squared range error of all neighboring anchor nodes.
The PSO algorithm provides better convergence than simulated annealing and ensures solution without being trapped into local minima.

YaoHung Wu et al. [20] proposed a distributed localization approach known as the Rectangle Overlapping Approach (ROA), which uses a moving beacon equipped with a GPS and a directional antenna. The positions can be determined using simple operations according to the current state of the moving beacon, including the rotation angle and position. The node positions can be determined accurately after the beacon operates along straight- line traverse routes.

JiaHuanxiang et al. [21] proposed a new localization method with mobile anchor node and genetic algorithm. It combines weighted centroid method with genetic algorithm. Initially, the mobile anchor node, which is equipped with GPS, was allowed to traverse around the entire sensing area. The unknown sensor nodes can obtain useful information for localization through mobile anchor node. Then, the initial coordinates of unknown sensor nodes are calculated by the weighted centroid method. Now, the initial position coordinates of the unknown sensor nodes are converged towards the actual coordinates. As the genetic algorithm is iterative - looped, the localization accuracy is improved to some extent.

Huan-qing Cui et al. [22] proposed a Weighted Centroid Localization method using three mobile beacons. These beacons preserve a special formation while traversing the network deployment area, and broadcast their positions periodically. The location unaware sensor nodes that are to be localized estimate the distances to these three beacons and use weighted centroid localization method to find its position. Through simulation results, this method was found superior to Weighted Centroid Localization method with a single mobile beacon as well as to Trilateration.

Zhen $\mathrm{Hu}$ et al. [23] proposed a Radio-Frequency (RF) based Mobile Anchor Centroid Localization method (MACL) for WSNs. In this method, a mobile anchor node moves in the sensing field and broadcast its current location periodically. Simulations and tests from an indoor deployment using the Cricket location system were used to investigate the localization accuracy of MACL. From the results of RF based MACL, it provides less computational complexity with low communication overhead, low cost, and flexible accuracy.

Lutful Karim et al. [24] proposed a Range-free Energy Efficient Localization Technique using Mobile Anchor (RELMA) especially for large scale WSNs to improve both accuracy and energy efficiency by minimizing the number of anchor nodes used. The RELMA Method 1 as well as RELMA_Method 2 has used the sensing range for each pair of nodes to communicate instead of the communication range to reduce the power consumptions of the nodes. The performance of RELMA_Method 1 and RELMA_Method 2 are compared only with the existing NeighboringInformation-Based Localization System (NBLS). Simulation results demonstrate the fact that RELMA_Method 1 and RELMA_Method 2 outperform NBLS in terms of localization accuracy as well as energy efficiency.

$\mathrm{Xu}$ Lei et al. [25] proposed a Mobile Anchor Assisted Localization Algorithm based on PSO (MAAAL_PSO) pertaining to adverse or dangerous application environments. The Region of Interest (ROI) is divided into grids and the mobile anchor deploys virtual anchors on the vertex of each grid. Based on this deployment, the node localization is 
converted into non-linear constrained optimization problem solved by PSO with the help of mobile anchor. After a few iterations, Performance evaluations demonstrate that this algorithm improves localization accuracy. It is also robust to the interference of environment noise.

The proposed approach in this paper is ant colony optimization with Mobile Anchor Positioning (ACO-MAP) for localization. Here the location of nodes is initially found using Mobile Anchor Positioning. Then ACO algorithm is applied over the results of MAP-M\&N. The observation is that, ACO-MAP approach estimated the location of the sensor nodes providing significant accuracy than MAP-M\&N.

\section{PROPOSED LOCALIZATION APPROACH}

The localization strategy used in this work can be visualized to work in two phases. In the first phase, a range-free algorithm namely Mobile Anchor Positioning with Mobile Anchor \& Neighbor (MAP-M\&N) is used for finding the location of the unknown sensor nodes. Since a range-free algorithm that offers coarse-grained accuracy is used, the obtained location will be just as an estimate. In the second phase (post optimization phase), evolutionary strategy namely Ant Colony Optimization with Mobile Anchor Positioning (ACO-MAP) is applied over MAP-M\&N for fine-tuning the results of the sensor nodes obtained from MAP-M\&N and thereby improving the localization accuracy.

\subsection{Mobile Anchor Positioning (MAP)}

The simulation environment is set-up as follows: The sensor nodes are randomly deployed in the sensing field. Mobile anchors are location aware nodes that move in the sensing field, fitted with GPS. As they move around the sensing field, they periodically broadcast messages containing their current location at fixed time interval to all the nodes, which are at a hearing distance from it. Such messages are known as beacons. The mobile anchors traverse around the field with a specific speed and their directions are set to change for every 10 seconds. All the nodes in the communication range of the mobile anchor will receive the beacons. A sensor node will collect all the beacons in its range and store it as a list. Communication range of the sensor node and the mobile anchor node are assumed as same. Once enough beacons are received and if a sensor node does not receive a beacon, which is at a distance greater than the already received ones, the localization begins at that particular node.

Assume that the sensor node has received and stored four beacons (locations of the mobile anchor) in its list $\left\{T_{1}, T_{2}, T_{3}\right.$, and T4 3 as shown below in Fig. 1. From the list, two beacons, which are farthest from each other, are chosen $\left(\mathrm{T}_{1}, \mathrm{~T}_{4}\right)$. These points are known as Beacon points. These two points are marked as the end of the sensor node's communication range since the sensor node has not received a beacon farther from this point. Hence $T_{1}$ and $T_{4}$ (Beacon points) represent either two positions of the same mobile anchor or positions of two different mobile anchors when they were at the end of the sensor node's communication range.

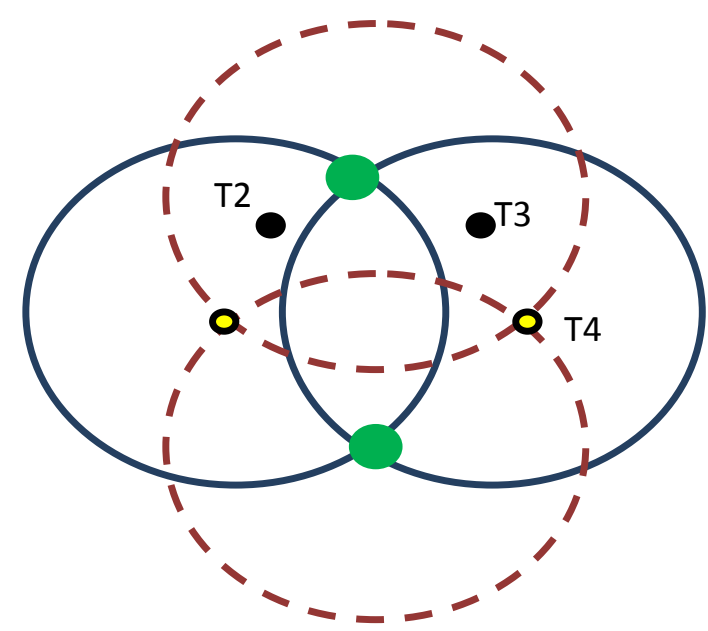

Possible locations of the sensor node

C

Beacon points

Beacon packets

Fig 1: Possible Locations of the Sensor Node

With these two Beacon points as centers and the communication range of a sensor node as radius, two circles are constructed (refer Fig. 1). Each circle represents the communication range of the mobile anchor, which has sent the beacon. The sensor node has to fall inside this communication range, as it has received the beacon. Since the sensor node has received packets either from both anchors or from the two positions of the same anchor, the node has to fall inside both the circles. Hence, it can be concluded that circles will intersect each other. The intersection points of both circles are determined $\left(\mathrm{S}_{1}, \mathrm{~S}_{2}\right)$. The intersection points are the possible locations of the sensor node. The reason is as follows: The two farthest points (Beacon points) are the end points of a sensor node's communication range.

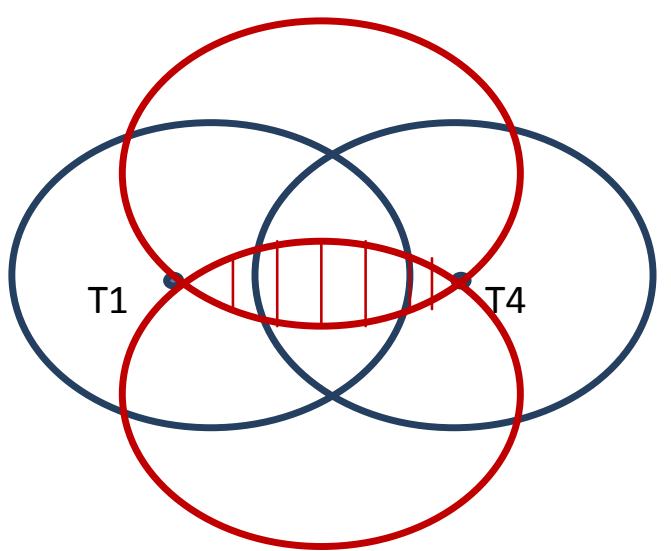

Figure 2: Shadow Area

The sensor node lies on the circumference of the other circle since it is the same with the other mobile anchor position. Therefore, the sensor node lies on the circumference of both the circles. The only points satisfying the above condition are the two intersection points. Hence, by means of Mobile Anchor Positioning, the location of the sensor node has been approximated to two locations. 


\subsubsection{Identifying the Sensor Locations using MAP with Mobile Anchor (MAP-M)}

The visitor list is searched after identifying the two possible positions i.e. the intersection points. If a node could hear around its range, there is a possibility of a beacon point which can be situated at a distance $r$ from one of the two possible locations. Thus, there is one point in the list, whose distance from one possible location is less than $\mathrm{r}$, and the distance from other possible location is greater than $r$, then the first possible location is chosen as the location of the sensor node. It is assumed that the communication range of a mobile anchor is $\mathrm{R}$. The MAP-M maintains the visitors list after receiving the beacon packets from the mobile anchor. The information from the visitor list is used to approximate the location of the sensor node.

Let the visitor list of a sensor node $\mathrm{S}$ consists of various location information represented as $\{\mathrm{T} 1, \mathrm{~T} 2 \ldots \mathrm{Tn}\}$. The beacon points are the two extreme points i.e., $\mathrm{T} 1$ and $\mathrm{Tn}$. Two circles with radius $\mathrm{R}$ and center $\mathrm{T} 1$ and $\mathrm{Tn}$ are constructed and their intersection points of two circles are found to be $S^{\prime}$ and $\mathrm{S}^{\prime \prime}$. If there is any $\mathrm{Ti}(2 \leq \mathrm{i} \leq \mathrm{n}-1)$, such that the distance between Ti and $\mathrm{S}^{\prime}$ is less than R and that between Ti and $\mathrm{S}^{\prime \prime}$ is greater than $\mathrm{R}$, then we can conclude the location of the sensor node is $\mathrm{S}^{\prime}$. This is because of the fact that the sensor node should lie inside the communication range of mobile anchor to receive the beacon packets. Consequently, the distance between the sensor node $\mathrm{S}$ and beacon packet $\mathrm{Ti}$ should be less than R. There is an area named as the shadow region, as shown in Fig. 2. If all the Beacon points lie inside this region, it is not possible to determine the location of the sensor as the shadow region comes under the range of both the intersection points. This could be explained by drawing two circles with $\mathrm{S}^{\prime}$ and $\mathrm{S}^{\prime \prime}$ as centre and the shadow region is the intersection of the two circles. Hence, in order to estimate the location of the sensor node there is a need that at least one of the beacon packets in the visitor list must lie outside the shadow region as in Fig.3.

Therefore, it is not possible to determine the location of the sensor node $\mathrm{S}$ using the available beacon packets, thus the node is made to wait until it gets further beacon packets. If no further beacons are obtained, then a single position of sensor node $\mathrm{S}$ cannot be obtained. The node will have two positions $\mathrm{S}^{\prime}$ and S". The method of Mobile Anchor Positioning - Mobile Anchor \& Neighbor (MAP-M\&N) is followed in order to overcome this problem.

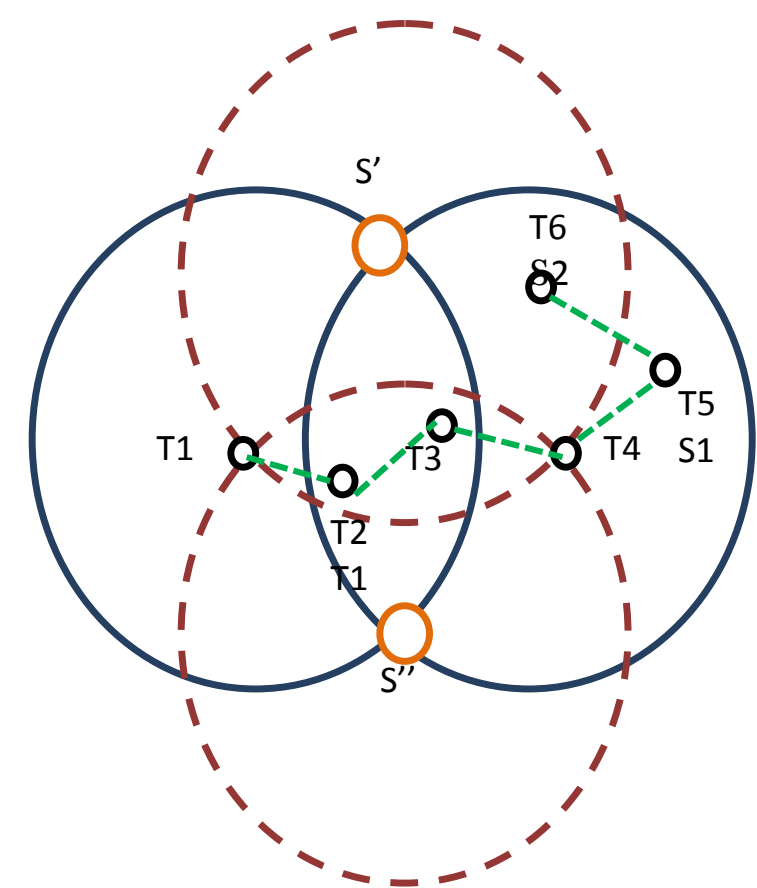

$\mathrm{S}$ ' and $\mathrm{S}$ ' indicate the possible locations of the Sensor node

O

Beacon packets

Figure 3: Node Seeking Information from Neighbor Sensors

\subsubsection{Forming additional Anchors and identifying the Sensor Locations using MAP with Mobile Anchor \& Neighbor (MAP-M\&N)}

The location estimation done for sensors using MAP-M method gives positions for few sensors and for the others, it gives two positions and so it is the responsibility of MAP$\mathrm{M} \& \mathrm{~N}$ method to produce outputs with a single position for each sensor. It is possible for the sensor nodes that have already determined their location to assist other nodes in determining their locations. As soon as the location is identified, the localized nodes start acting like anchors. They embed their calculated location inside the packet and then broadcast the beacons. Nodes, which are at its hearing range and waiting for additional beacons to finalize their location, can make use of these beacons. However, if the sensor node has determined its location, it simply discards the beacon packet. As a consequence, by using MAP-M\&N, the cost of movement of the mobile anchor can be reduced.

The localization steps for finding the location of the sensors in the field using MAP $-\mathrm{M} \& \mathrm{~N}$ algorithm are as follows:

1. Deploy hundred sensor nodes randomly in the 1000 $\mathrm{m} \times 1000 \mathrm{~m}$ area of the sensing field in the simulation environment and deploy three anchor nodes i.e sensor nodes fit with GPS

2. The assumption made is that the anchor nodes move in the entire sensing field based on a movement file which is fed as input to ns-2 simulator. The anchor nodes periodically broadcast their location packets known as beacon packets, while on the move through the sensing field. 
3. Every sensor node maintains a visitor list containing beacon packets based on the information obtained from anchors.

4. The sensor nodes can identify the farthest beacon packets and chooses those beacon packets as beacon points.

5. With those two beacon points as the centers and the communication range of a sensor node as radius, two circles are constructed and the intersection points are found.

6. Sensor nodes try to identify its position out of the two intersection points. Here, at least one of the beacon points in the visitor list must lie outside the shadow region or based on the beacon points obtained from neighbor nodes.

7. The approximate location for each of the sensor nodes is estimated using the MAP-M\&N method.

\subsection{Ant Colony Optimization with Mobile Anchor Positioning (ACO-MAP)}

The Ant Colony Optimization (ACO) algorithm takes the results of MAP-M\&N as the input. The localization steps followed by using ACO Algorithm [26] are listed below:

1. The algorithm takes the results of Mobile Anchor Positioning as its input. The results of MAP-M\&N, giving the approximate solution of the location of each sensor at each specified time instance is given as the input to the post optimization method.

2. Let each sensor's $x$ and $y$ co-ordinates at different instances of time be $\left(x_{1}, y 1\right),\left(x_{2}, y 2\right) \ldots\left(x_{n}, y_{n}\right)$, where $\mathrm{n}$ denotes the number of sensor nodes. Each of these positions is considered as a separate ant. Hence producing as much of ant as that of the approximate positions found at regular intervals.

3. The first ant moves randomly until it finds the food source, then it returns to the nest, laying a pheromone trail. Once the ant's path is complete, by applying the local update rule it updates its pheromone value.

4. Other ants follow one of the paths at random, also laying pheromone trails. Since the ants on the shortest path lay pheromone trails faster, this path gets reinforced with more pheromone, making it more appealing to future ants.

5. When all ants have completed their path, it modifies the pheromone value by global update rule again.

6. The ants become increasingly likely to follow the shortest path since it is constantly reinforced with a larger amount of pheromones. The pheromone trails of the longer paths evaporate.

7. When the ants select the path again, it will tend to choose the path of higher pheromone, and release the pheromone.

8. After much iteration, there will be a pheromone on a path much more than the other path, this path is the optimal path.

9. Repeat the procedure until the stopping criteria is met.

Stopping criteria $=$ Maximum iterations or Profit value
Maximum iteration $=$ arbitrarily chosen as 100.

\section{SIMULATION RESULTS}

The following parameters namely Number of Mobile Anchors, Speed of Mobile Anchors, Number of Sensor Nodes and Execution time are varied and the results were analyzed for each of the parameter variation as shown in Table 1.

Table 1: Simulation Settings

\begin{tabular}{|l|l|}
\hline Parameter Description & Value \\
\hline Number of Sensor Nodes & 100 \\
\hline Area of the Sensing Field & $1000 \mathrm{X} 1000 \mathrm{~m}^{2}$ \\
\hline Number of Mobile Anchors & 3 \\
\hline Speed of Mobile Anchors & $100 \mathrm{~m} / \mathrm{s}$ \\
\hline $\begin{array}{l}\text { Time interval between } \\
\text { successive Anchors }\end{array}$ & $1 \mathrm{~s}$ \\
\hline Execution time & $500 \mathrm{~s}$ \\
\hline Transmission range & $250 \mathrm{~m}$ \\
\hline Routing Protocol & AODV \\
\hline MAC Protocol & IEEE 802.11 \\
\hline
\end{tabular}

With the simulation settings of ns-2 simulator as shown in Table 1 , the results were analyzed by comparing the performance of the proposed Ant Colony Optimization with MAP (ACO-MAP) approach with regard to MAP-M\&N.

\subsection{Metric used to determine Localization Accuracy}

The metric that is used to evaluate the accuracy in localization process is Root Mean Square Error (RMSE). Equation (1) states the RMSE formula for proposed evolutionary approach as,

$$
R M S E=\sqrt{\sum_{i=1}^{n} \frac{\left(x_{a c t(i)}-x_{o b t(i)}\right)^{2}+\left(y_{a c t(i)}-y_{o b t(i)}\right)^{2}}{N}}
$$

Where, $X_{a c t(i)}, y_{a c t}(i)$ - represent the actual values of $\mathrm{x}$ and $\mathrm{y}$ coordinates of the sensor nodes, $x_{o b t(i)}, y_{o b t(i)}$ - represent the obtained values $\mathrm{x}$ and $\mathrm{y}$ coordinates of the sensor nodes and $N$-represents the total number of Localized nodes.

\subsubsection{Comparison of RMSE obtained using ACO- $M A P$ and $M A P-M \& N$}

The localization accuracy of sensor nodes is evaluated based on the positional error minimization. The Root Mean Square Error is calculated for both ACO-MAP and MAP-M\&N approaches pertaining to every ten nodes scenario as listed in Table 2.

The Table 2 shows the RMSE analysis of the two algorithms namely, MAP-M\&N and ACO-MAP corresponding to 10, 20, 30 etc. up to 100 nodes scenario on an average clearly illustrates the fact that RMSE gets drastically reduced when ACO is used along with MAP (ACO-MAP) when compared to MAP-M\&N algorithm alone for estimating the location of sensors. 
Table 2: RMSE Calculation for MAP-M\&N algorithm and proposed ACO-MAP

\begin{tabular}{|l|l|l|}
\hline $\begin{array}{l}\text { No. of } \\
\text { Nodes }\end{array}$ & $\begin{array}{l}\text { RMSE obtained } \\
\text { by MAP-M\&N }\end{array}$ & $\begin{array}{l}\text { RMSE obtained } \\
\text { By ACO-MAP }\end{array}$ \\
\hline 10 & 237.75 & 7.49 \\
\hline 20 & 330.07 & 7.87 \\
\hline 30 & 252.72 & 8.60 \\
\hline 40 & 331.93 & 8.26 \\
\hline 50 & 345.01 & 7.59 \\
\hline 60 & 232.80 & 8.56 \\
\hline 70 & 387.03 & 8.41 \\
\hline
\end{tabular}

\begin{tabular}{|l|l|l|}
\hline 80 & 222.61 & 8.89 \\
\hline 90 & 270.92 & 8.64 \\
\hline 100 & 261.98 & 8.72 \\
\hline
\end{tabular}

This observation shown in Table 2 has been graphically displayed in Fig.4 as shown above where, the $\mathrm{x}$-axis indicates the number of nodes and the $y$-axis indicates the corresponding value of RMSE. It can be clearly noted from Fig.4 that the values of RMSE obtained using ACO-MAP approach corresponding to $10,20,30$ etc. up to 100 nodes scenario on an average reduces significantly when compared to MAP-M\&N

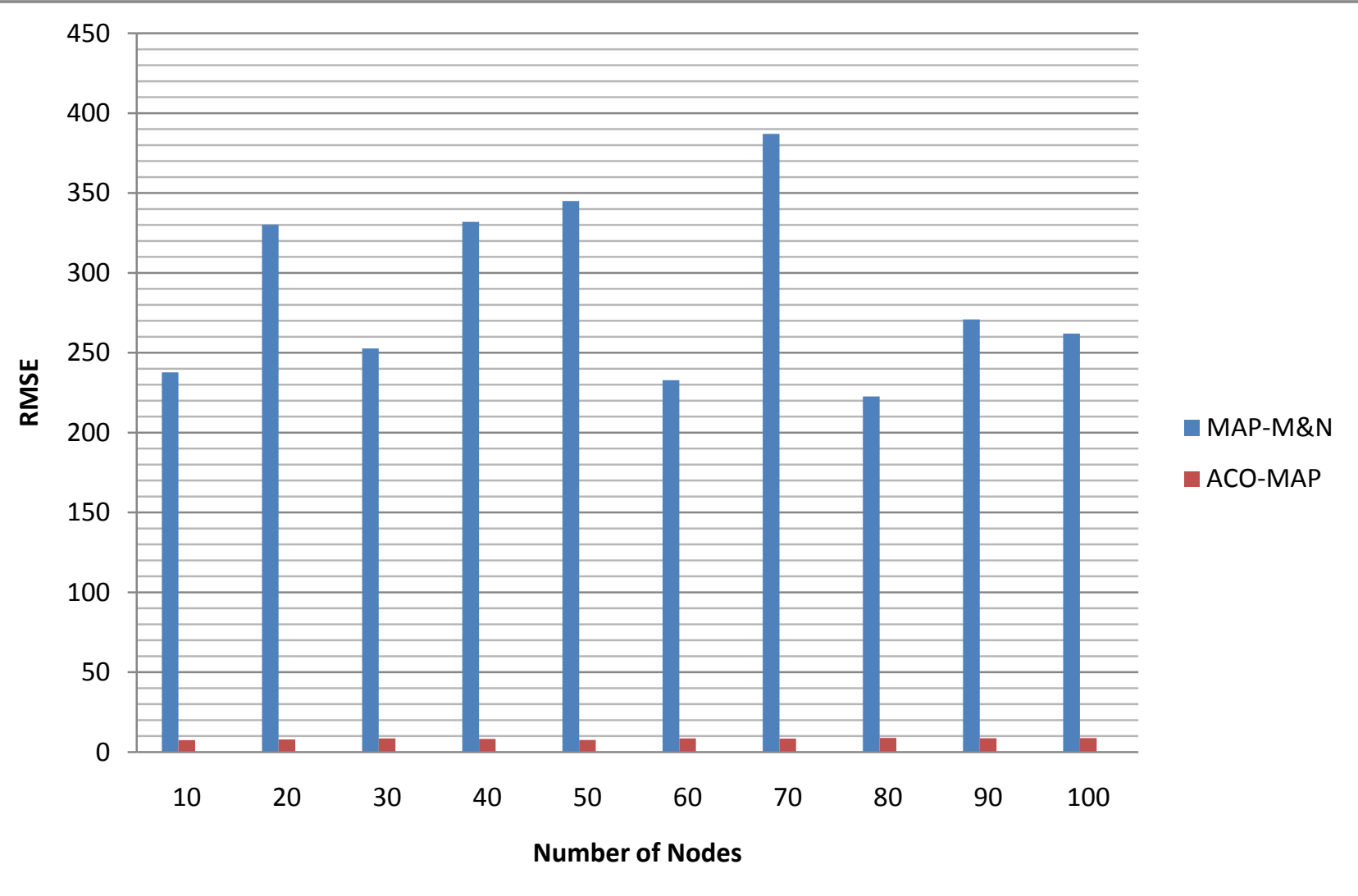

Figure 4: Comparison Graph for RMSE of ACO-MAP versus MAP-M\&N algorithm

\section{CONCLUSION}

Mobile Anchor Positioning with Mobile Anchor \& Neighbor (MAP-M\&N) algorithm uses range-free localization method that does not involve usage of any hardware. The percentage of localized nodes is high which indicates that MAP-M\&N is suitable for localization. Since this method does not provide fine-grained accuracy in localization, optimization techniques are applied over the results of MAP-M\&N. By considering hundred unknown sensor nodes as inputs and observing the simulation results based on RMSE, Ant colony optimization with Mobile Anchor Positioning (ACO-MAP) algorithm minimizes the localization error significantly by $96.9 \%$ on an average when compared to MAP-M\&N algorithm.

Consequently it is obvious that ACO-MAP evolutionary approach minimizes the error in localization much better than using MAP-M\&N algorithm. To further minimize the localization error, hybrid optimization algorithm such as Simulated Annealing (SA) may be combined with Ant colony optimization with Mobile Anchor Positioning (ACO-MAP) algorithm. The localization error of the new hybrid evolutionary algorithm can be compared with Ant colony optimization (ACO-MAP) to validate its performance. The future work may be applying meta-heuristic optimization techniques such as artificial bee colony $(\mathrm{ABC})$ algorithm with mobile anchor positioning to further minimize the error in localization of a wireless sensor network. 


\section{REFERENCES}

[1] I.F.Akyildiz, W.Su, Y. Sankarasubramanium, and E.cayirci, "Wireless Sensor Networks: A Survey," IEEE Computer., Vol. 38, Issue 4, pp.393 -422, 2002.

[2] Jonathan Bachrach and Christopher Taylor, "Localization in Sensor Networks," Chapter 9, "Handbook of Sensor Networks: Algorithms and Architectures," by Ivan Stojmenovic, pp. 277-297, 2006.

[3] Guoqiang Mao, Barıs, Fidan, and Brian D.O. Anderson, "Wireless Sensor Networks Localization Techniques," Science Direct, Computer Networks 51, pp. 2599-2533, 2007.

[4] GuibinZhu,Qiuhua Li, PengQuan, and Jiuzhi Ye, "A GPS- free Localization scheme for Wireless Sensor Networks," $12^{\text {th }}$ IEEE International Conference on Communication Technology (ICCT 2010), pp. 401-404, Nov 2010.

[5] Chaczko Zenon, Klempous Ryszard, Nikodem Jan, and Nikodem Michal, "Methods of Sensors Localization in Wireless Sensor Networks,"14 ${ }^{\text {th }}$ Annual International Conference and Workshops on Engineering on Computer based Systems (ECBS 2007), pp. 145-152, Mar 2007.

[6] Hoang Q.T., Le T.N., and Yoan Shin, "An RSS comparison based Localization in Wireless Sensor Networks," $8^{\text {th }}$ workshop on Positioning Navigation and communication (WPNC 2011), pp.116-121, April 2011.

[7] Pengfei Peng, HaoLuo, Zhong Liu, and XiongweiRen, "A cooperative target location algorithm based on Time Difference of Arrival in Wireless Sensor Networks," International Conference on Mechatronics and Automation (ICMA 2009), pp. 696-701, Aug 2009.

[8] GuoweiShen, Zetik R, Honghui Yan, Hirsch O., and Thoma R.S., "Time of Arrival Estimation for RangeBased Localization in UWB Sensor Networks," IEEE International Conference on Ultra-Wideband (ICUWB 2010), Vol. 2, pp. 1-4, Sep 2010.

[9] Yanping Zhu, Daqing Huang, and Aimin Jiang, "Network Localization using Angle of Arrival," IEEE International Conference on Electro/Information Technology (EIT 2008), pp. 205-210, May 2008.

[10] Ganggang Yu, Fengqi Yu, and Lei Feng, "ALocalization Algorithm using a Mobile Anchor Node under Wireless Channel," IEEE International Conference on Robotics and Biomimetics, Dec 2007.

[11] Binwei Deng, Guangming Huang, Lei Zhang, and Hao Liu, "Improved Centroid Localization Algorithms in WSNs," $3^{\text {rd }}$ International Conference on Intelligent System and Knowledge Engineering (ISKE 2008), Vol. 1, pp. 1260-1264, Nov 2008.

[12] Zhang Zhao-yang, Gou Xu, Li Ya-peng, and Shan-shan Huang, "DV Hop based Self-Adaptive Positioning in Wireless Sensor Networks," $5^{\text {th }}$ International Conference on Wireless Communications, Networking and Mobile Computing (WiCom 2009), pp. 1-4, Sept. 2009.

[13] Patro, R.K, "Localization in Wireless Sensor Network with Mobile Beacons," $23^{\text {rd }}$ IEEE convention of Electrical and Electronics Engineers Israel, pp. 22-24, Sept. 2004.
[14] Qingguo Zhang, Jinghua Wang, Cong Jin, and QingjiangZeng, "Localization Algorithm for Wireless Sensor Network based on Genetic Simulated Annealing Algorithm," IEEE International conference on Wireless Communications, Networking and Mobile Computing ,2008

[15] W-H Liao, Y.C.Lee, and S.P. Kedia, "Mobile Anchor Positioning of Wireless Sensor Networks," IET communications, Vol. 5, Issue 7, pp.914-921, 2011.

[16] Kuo-Feng Ssu, Chia-Ho Ou, and Hewijin Christine Jiau, "Localization with Mobile Anchor Points in Wireless Sensor Networks,'IEEE Transactions on Vehicular Technology, Vol. 54, No. 3, May 2005.

[17] Baoli Zhang, Fengqi Yu, and Zushengzhang, "An improved Localization Algorithm for Wireless Sensor Network using a Mobile Anchor Node," Asia-Pacific Conference on Information Processing, 2009.

[18] Wenwen Li and Wuneng Zhou, "Genetic Algorithm Base Localization Algorithm for Wireless Sensor Networks," $7^{\text {th }}$ International Conference on Natural Computation (ICNC 11), pp. 2096-2099, July 2011.

[19] Gopakumar.A and Jacob.L, "Localization in Wireless Sensor Networks using Particle Swarm Optimization," IET International Conference on Wireless, Mobile and Multimedia Networks, pp. 227-230, 2008.

[20] YaoHungWu and WeiMeiChen, "Localization of Wireless Sensor Networks using a Moving Beacon with a Directional Antenna[C]," Proc. of the $11^{\text {th }}$ International Conference on High Performance Computing and Communications, Korea, pp. 237-242, 2009.

[21] JiaHuanxiang and Wang Yong, "Localization Algorithm for Mobile Anchor node based on Genetic Algorithm in Wireless Sensor Network," Int. Conf. on Intelligent Computing and Integrated $\backslash$ Systems(ICISS),pp.4044,2010 .

[22] Huan-Qing Cui , Ying-Long Wang, Jia-Liang Lv, and Yu-Ming Mao, " Three-Mobile Beacon Assisted Weighted Centroid Localization Method in Wireless Sensor Networks," Proc. of $2^{\text {nd }}$ IEEE Int. Conf. on Software Engineering and Service Science (ICSESS), pp. 308-311, July 2011.

[23] Zhen Hu , Dongbing Gu, Zhengxun Song , and Hongzuo $\mathrm{Li}$, "Localization in Wireless Sensor Networks using a Mobile Anchor Node," Proc. of IEEE / ASME International Conference on Advanced Intelligent Mechatronics (ICAIM), pp. 602-607, July 2008.

[24] Lutful Karim, Nidal Nasser, and Tarek El Salti, "RELMA: A Range free Localization Approach using Mobile Anchor Node for Wireless Sensor Networks," Proc. of IEEE Globecom, 2010.

[25] Xu Lei, Zhang Huimin, and Shi Weiren, "Mobile Anchor assisted Node Localization in Sensor Networks based on Particle Swarm Optimization,” Proc. of IEEE WiCom, 2010.

[26] Fu Qin, Chen Wei, Liu Kezhong, "Node Localization with a Mobile Beacon based on Ant Colony Algorithm in Wireless Sensor Networks ", Proc. of IEEE International Conference on Communications and Mobile Computing (CMC), Shen Zhen, Vol.3, PP. 303-307, April 2010. 